\title{
NOETHER-LASKER DECOMPOSITION OF COHERENT ANALYTIC SUBSHEAVES
}

\author{
BY \\ YUM-TONG SIU
}

In this paper we develop the theory of Noether-Lasker decomposition of coherent analytic subsheaves as an analogue of the algebraic Noether-Lasker decomposition of ideals in Noetherian rings. The decomposition can be described as follows: Suppose $\mathscr{S}$ is a coherent analytic subsheaf of a coherent analytic sheaf $\mathscr{T}$ on a complex space $(X, \mathcal{O})$ in the sense of Grauert. For every point $x$ of $X$, $\mathscr{S}_{x}$ as an $\mathcal{O}_{x}$-submodule of $\mathscr{T}_{x}$ has a Noether-Lasker decomposition into primary $\mathcal{O}_{x}$-submodules of $\mathscr{T}_{x}$. The radicals of these primary submodules are prime ideals of $\mathcal{O}_{x}$ which define subvariety-germs of $X$ at $x$. These subvariety germs are pieced together to form global irreducible subvarieties of $X$ which we call associated subvarieties of $\mathscr{S}$. A coherent subsheaf of $\mathscr{T}$ which has only one associated subvariety is called primary. We prove that every coherent analytic subsheaf can be represented as the intersection of "locally finite" primary subsheaves. This representation is what we call the Noether-Lasker decomposition of the coherent analytic subsheaf. If $(X, \mathcal{O})$ is Stein, then a coherent analytic proper subsheaf $\mathscr{S}$ of a coherent analytic sheaf $\mathscr{T}$ is primary if and only if $\Gamma(X, \mathscr{S})$ is a primary submodule of the $\Gamma(X, \mathcal{O})$-module $\Gamma(X, \mathscr{T})$.

The Noether-Lasker decomposition of subsheaves is derived from the gapsheaf theory of Thimm [4]. In part I of this paper we give an exposition of Thimm's theory of gap-sheaves by sheaf-theoretical methods. In part II of this paper we establish the Noether-Lasker decomposition of coherent analytic subsheaves.

Notations. All complex spaces in this paper are in the sense of Grauert $[1, \S 1]$. Suppose $(X, \mathcal{O})$ is a complex space. A holomorphic function on $(X, \mathcal{O})$ is an element of $\Gamma(X, \mathcal{O})$. A holomorphic function $f$ vanishes at a point $x$ of $X$ if the germ of $f$ at $x$ is not a unit in $\mathcal{O}_{x}$. A subvariety in $X$ is a set which locally is the set of points where a finite number of locally defined holomorphic functions vanish. The ideal-sheaf of a subvariety $Y$, denoted by Id $Y$, is the sheaf of germs of holomorphic functions vanishing at every point of $Y$. A complex space $(Z, \mathscr{H})$ is a subspace of $(X, \mathcal{O})$ if $Z$ is a subvariety of $X$ and there exists a coherent ideal-sheaf $\mathscr{I}$ on $X$ such that $\mathscr{H}=(\mathscr{O} / \mathscr{I}) \mid Z$ and $\left\{z \mid z \in X, \mathscr{I}_{z} \neq \mathcal{O}_{z}\right\}=Z$. A module-sheaf on $(X, \mathcal{O})$ is an analytic subsheaf of $\mathcal{O}^{p}$ for some $p$. If $\mathscr{A}$ is an ideal-sheaf on $(X, \mathcal{O})$, then $\sqrt{ } \mathscr{A}$ is the ideal-sheaf defined by $(\sqrt{ } \mathscr{A})_{x}=\sqrt{ } \mathscr{A}_{x}$, where $\sqrt{ } \mathscr{A}_{x}$ is the radical of the ideal $\mathscr{A}_{x}$ in $\mathcal{O}_{x}$.

Received by the editors March 14, 1967 and, in revised form, August 24, 1967. 
Suppose that $\mathscr{R}$ and $\mathscr{S}$ are analytic subsheaves of an analytic sheaf $\mathscr{T}$ and $\mathscr{A}$ is an ideal-sheaf on a complex space $(X, \mathcal{O})$. Denote by $\mathscr{R}: \mathscr{S}$ the ideal-sheaf defined as follows: for $x \in X, f \in(\mathscr{R}: \mathscr{S})_{x}$ if and only if $f \in \mathcal{O}_{x}$ and $f \mathscr{S}_{x} \subset \mathscr{R}_{x}$. Denote by $(\mathscr{S}: \mathscr{A})_{\mathscr{T}}$ or simply by $\mathscr{S}: \mathscr{A}$ the subsheaf of $\mathscr{T}$ defined as follows: for $x \in X, s \in(\mathscr{S}: \mathscr{A})_{x}$ if and only if $s \in \mathscr{T}_{x}$ and $\mathscr{A}_{x} s \subset \mathscr{S}_{x}$. If $\mathscr{R}, \mathscr{S}, \mathscr{T}$, and $\mathscr{A}$ are coherent, then $\mathscr{R}: \mathscr{S}$ and $\mathscr{S}: \mathscr{A}$ are coherent. If $s \in \Gamma(X, \mathscr{T})$ and $f \in \Gamma(X, \mathcal{O})$, then $\mathscr{R}: s$ denotes $\mathscr{R}: \mathcal{O}_{s}$ and $(\mathscr{S}: f)_{\mathscr{T}}$ or simply $\mathscr{S}: f$ denotes $(\mathscr{P}: \mathcal{O} f)_{\mathscr{T}}$.

Suppose $X$ and $Y$ are two complex spaces, $\mathscr{T}$ is an analytic sheaf on $X$, and $\pi: X \rightarrow Y$ is a holomorphic map (i.e. a morphism of ringed spaces). Then $R^{0} \pi(\mathscr{T})$ denotes the zeroth direct image of $\mathscr{T}$ under $\pi$.

Suppose $x=\left(x_{1}, \ldots, x_{n}\right) \in C^{n}$ and $r_{1}, \ldots, r_{n}$ are positive numbers. Then $\Delta\left(x ; r_{1}, \ldots, r_{n}\right)$ denotes the polydisc $\left\{\left(z_{1}, \ldots, z_{n}\right) \in C^{n}|| z_{i}-x_{i} \mid<r_{i}, 1 \leqq i \leqq n\right\}$. Suppose $\mathscr{F}$ is a sheaf on a topological space $E, x \in E, U$ is an open neighborhood of $x$ in $E$, and $s \in \Gamma(U, \mathscr{F})$. Then $s_{x}$ denotes the germ of $s$ at $x$ and $\mathscr{F}_{x}$ denotes the stalk of $\mathscr{F}$ at $x$. If $f$ is a (complex-valued) function on $E$, then $f_{x}$ denotes the germ of $f$ at $x$.

Suppose $R$ is a Noetherian ring and $E$ is an $R$-submodule of a finitely generated $R$-module $F$. Then $P(E, F)$ denotes the set of all associated nonunit prime ideals in the Noether-Lasker decomposition of $E$ as a submodule of $F$.

\section{Gap-sheaves.}

Definition 1. Suppose $\mathscr{S}$ is an analytic subsheaf of an analytic sheaf $\mathscr{T}$ on a complex space $(X, \mathcal{O})$ and $\rho$ is a nonnegative integer. The $\rho$ th gap-sheaf of $\mathscr{S}$ in $\mathscr{T}$, denoted by $\mathscr{S}_{[0] \mathscr{F}}$ or simply $\mathscr{S}_{[0]}$, is the analytic subsheaf of $\mathscr{T}$ defined as follows: for $x \in X, s \in\left(\mathscr{S}_{[\rho]}\right)_{x}$ if and only if there exist an open neighborhood $U$ of $x$ in $X$, a subvariety $A$ in $U$ of dimension $\leqq \rho$, and $t \in \Gamma(U, \mathscr{T})$ such that $t_{x}=s$ and $t_{y} \in \mathscr{S}_{y}$ for $y \in U-A$. Denote by $E(\mathscr{S}, \mathscr{T})$ the set $\left\{x \mid x \in X, \mathscr{S}_{x} \neq \mathscr{T}_{x}\right\}$ and $E^{o}(\mathscr{S}, \mathscr{T})$ denotes $E\left(\mathscr{S}, \mathscr{S}_{[\rho]}\right)$.

Definition 2. Suppose $\mathscr{S}$ is an analytic subsheaf of an analytic sheaf $\mathscr{T}$ on a complex space $(X, \mathcal{O})$ and $A$ is a subvariety of $X$. Then the gap-sheaf of $\mathscr{S}$ in $\mathscr{T}$ with respect to $A$, denoted by $\mathscr{S}[A]_{\mathscr{F}}$ or simply $\mathscr{S}[A]$, is the analytic subsheaf of $\mathscr{T}$ defined as follows: for $x \in X, s \in \mathscr{S}[A]_{x}$ if and only if there exist an open neighborhood $U$ of $x$ in $X$ and $t \in \Gamma(U, \mathscr{T})$ such that $t_{x}=s$ and $t_{y} \in \mathscr{S}_{y}$ for $y \in U-A$.

THEOREM 1. Suppose $\mathscr{S}$ is a coherent analytic subsheaf of a coherent analytic sheaf $\mathscr{T}$ on a complex space $(X, \mathcal{O})$ and $A$ is a subvariety of $X$. Then

$$
\mathscr{S}[A]=\bigcup_{n=1}^{\infty}\left(\mathscr{S}: \mathscr{A}^{n}\right)_{\mathscr{T}},
$$

where $\mathscr{A}$ is the ideal-sheaf of $A$, and hence is coherent.

Proof. Let $\mathscr{F}=\bigcup_{n=1}^{\infty}\left(\mathscr{S}: \mathscr{A}^{n}\right)_{\mathscr{T}} \subset \mathscr{T}$. $\mathscr{F}$ is coherent, because it is the union of an increasing sequence of coherent subsheaves of a coherent sheaf [1, Satz 8, §2].

Suppose $s \in \mathscr{S}[A]_{x}$ for some $x \in X$. Then there exist an open neighborhood $U$ of 
$x$ in $X$ and $t \in \Gamma(U, \mathscr{T})$ such that $t_{x}=s$ and $t_{y} \in \mathscr{S}_{y}$ for $y \in U-A$. Let $\mathscr{B}=(\mathscr{S} \mid U): t$. $E(\mathscr{B}, \mathcal{O} \mid U) \subset A \cap U$. By Hilbert Nullstellensatz [2, III.A.7] $\mathscr{A}_{x}^{n} \subset \mathscr{B}_{x}$ for some $n$. $s \mathscr{A}_{x}^{n} \subset s \mathscr{B}_{x} \subset \mathscr{S}_{x}, s \in \mathscr{F}$.

Suppose $s \in \mathscr{F}_{x} . s \in\left(\mathscr{S}: \mathscr{A}^{n}\right)_{x}$ for some $n$. There is an open neighborhood $U$ of $x$ and $t \in \Gamma(U, \mathscr{T})$ such that $t_{x}=s$ and $t\left(\mathscr{A}^{n} \mid U\right) \subset \mathscr{S} \mid U$. For $y \in U-A, \mathscr{A}_{y}^{n}=\mathcal{O}_{y}$. Hence $t_{y} \in \mathscr{S}_{y}$. $\mathscr{F}=\mathscr{S}[A]$. Q.E.D.

The following lemma is a particular case of [1, Hauptsatz I, §6] and it can be proved in a very elementary way.

Lemma 1. Suppose $X$ and $Y$ are complex spaces, $\mathscr{F}$ is a coherent sheaf on $X$, and $\pi: X \rightarrow Y$ is a proper nowhere degenerate holomorphic map, then $R^{0} \pi(\mathscr{F})$ is coherent.

THEOREM 2. Suppose $\mathscr{S}$ is a coherent analytic subsheaf of a coherent analytic sheaf $\mathscr{T}$ on a complex space $(X, \mathscr{H})$ and $\rho$ is a nonnegative integer. Then $E^{\rho}(\mathscr{S}, \mathscr{T})$ is locally contained in a subvariety of dimension $\leqq \rho$, i.e. for every $x \in X$ there exist an open neighborhood $U$ of $x$ in $X$ and a subvariety $A$ in $U$ of dimension $\leqq \rho$ such that $E^{\circ}(\mathscr{S}, \mathscr{T}) \cap U \subset A$.

Proof. Since the theorem is local in nature, we can suppose without loss of generality that $(X, \mathscr{H})$ is a subspace of an open subset $G$ of $C^{n}$. Let $\mathcal{O}$ be the structure sheaf of $G$ and $\tilde{\mathscr{S}}$ and $\tilde{\mathscr{T}}$ be the trivial extensions of $\mathscr{S}$ and $\mathscr{T}$ on $G$ respectively. We can further suppose without loss of generality that we have a sheaf-epimorphism $\lambda: \mathcal{O}^{p} \rightarrow \tilde{\mathscr{T}}$ on $G$. Let $\mathscr{M}=\lambda^{-1}(\tilde{\mathscr{S}})$. Then $E^{\rho}(\mathscr{S}, \mathscr{T})=E^{\rho}\left(\mathscr{M}, \mathcal{O}^{p}\right)$. Hence we need only prove that

for every coherent module-sheaf $\mathscr{M} \subset \mathscr{O}^{p}$ on an open subset $G$ of $C^{n}, E^{\rho}\left(\mathscr{M}, \mathcal{O}^{p}\right)$ is locally contained in a subvariety of dimension $\leqq \rho$.

We fix $\rho$ and prove (1) by induction on $n$. For $n \leqq \rho$, (1) is trivially true. Now suppose (1) is true when $n$ is replaced by $n-1$. We are going to prove by induction on $p$ that (1) is true when $n$ is unreplaced.

(a) $p=1$. Because of the local nature of (1) we can suppose that $G$ is connected. If $\mathscr{M}=0$, then (1) is trivial. So we can suppose $\mathscr{M} \neq 0 . E(\mathscr{M}, \mathcal{O})$ is a proper subvariety of $G$. Take $x \in G$. We want to prove that $E^{o}(\mathscr{M}, \mathcal{O})$ is locally contained in a subvariety of dimension $\leqq \rho$ at $x$. If $x \notin E(\mathscr{M}, \mathcal{O})$, then it is obviously true. So we suppose $x \in E(\mathscr{M}, \mathcal{O})$. There is a nonzero holomorphic function $\varphi$ on some open neighborhood $U$ of $x$ such that $\varphi$ vanishes on $E(\mathscr{M}, \mathcal{O}) \cap U$. Without loss of generality we can suppose that $U$ is a polydisc $\Delta\left(x ; r_{1}, \ldots, r_{n}\right)$ and the projection $\pi: Y \rightarrow \Delta\left(\pi(x) ; r_{1}, \ldots, r_{n-1}\right)$ defined by $\pi\left(z_{1}, \ldots, z_{n}\right)=\left(z_{1}, \ldots, z_{n-1}\right)$, where $Y=\{y \mid y \in U, \varphi(y)=0\}$, makes $Y$ an analytic cover over $\Delta\left(\pi(x) ; r_{1}, \ldots, r_{n-1}\right)$ [2, III.B.3].

By Hilbert Nullstellensatz, after shrinking $U$ we can suppose without loss of generality that $\varphi^{m} \in \Gamma(U, \mathscr{M})$ for some $m . \mathscr{Q}=\left(\mathscr{M} \mid \mathcal{O} \varphi^{m}\right) \mid Y$ is a coherent analytic 
sheaf on the complex space $(Y, \mathscr{K})$, where $\mathscr{K}=\left(\mathcal{O} / \mathcal{O} \varphi^{m}\right) \mid Y . \quad U \cap E^{\rho}(\mathscr{M}, \mathcal{O})$ $=E^{\rho}(\mathscr{Q}, \mathscr{K})$. Let $\mathscr{A}=R^{0} \pi(\mathscr{Q})$ and $\mathscr{B}=R^{0} \pi(\mathscr{K})$.

By Lemma $1, \mathscr{A}$ is a coherent analytic subsheaf of the coherent analytic sheaf $\mathscr{B}$ on $\Delta\left(\pi(x) ; r_{1}, \ldots, r_{n-1}\right)$.

If $y \in E^{\rho}(\mathscr{Q}, \mathscr{K})$, then there exist a subvariety $A$ of dimension $\leqq \rho$ in an open neighborhood $B$ of $y$ in $Y$ and $t \in \Gamma(B, \mathscr{K})$ such that $t_{z} \in \mathscr{Q}_{z}$ for $z \in B-A$ and $t_{y} \notin \mathscr{Q}_{y}$. Let $\pi^{-1}(\pi(y))=\left\{y^{0}, \ldots, y^{l}\right\}$, where $y^{0}=y$, and $B^{i}$ be disjoint open neighborhoods of $y^{i}, 0 \leqq i \leqq l$, in $Y$, such that $B^{0} \subset B$. Since $\varphi$ is proper, there is an open neighborhood $C$ of $\pi(y)$ in $\Delta\left(\pi(x) ; r_{1}, \ldots, r_{n-1}\right)$ such that $\pi^{-1}(C) \subset \bigcup_{i=0}^{l} B^{4}$. Define $t^{*} \in \Gamma\left(\pi^{-1}(C), \mathscr{K}\right)$ as follows: $t^{*}\left|\pi^{-1}(C) \cap B^{0}=t\right| \pi^{-1}(C) \cap B^{0}$ and $t^{*} \mid \pi^{-1}(C) \cap B^{i}=0$ for $1 \leqq i \leqq l$. $t^{*}$ induces $t^{\prime} \in \Gamma(C, \mathscr{B}) . t_{z}^{\prime} \in \mathscr{A}_{z}$ for $z \in C-\pi(A)$ and $t_{\pi(y)}^{\prime} \notin \mathscr{A}_{\pi(y)}$. Since $C \cap \pi(A)$ is a subvariety of dimension $\leqq \rho$ in $C, \pi(y) \in$ $E^{\rho}(\mathscr{A}, \mathscr{B})$. Since $y$ is an arbitrary point in $E^{\rho}(\mathscr{Q}, \mathscr{K}), E^{\rho}(\mathscr{Q}, \mathscr{K}) \subset \pi^{-1}\left(E^{\rho}(\mathscr{A}, \mathscr{B})\right)$. Let $0<s_{i}<r_{i}, 1 \leqq i \leqq n-1$, and $\mathscr{R}$ be the structure sheaf of $D=\Delta\left(\pi(x) ; s_{1}, \ldots, s_{n-1}\right)$. Then there is a sheaf-epimorphism $\eta: \mathscr{R}^{q} \rightarrow \mathscr{B} \mid D$.

$E^{o}(\mathscr{A}, \mathscr{B}) \cap D=E^{o}\left(\eta^{-1}(\mathscr{A} \mid D), \mathscr{R}^{q}\right)$. By induction hypothesis $E^{o}\left(\eta^{-1}(\mathscr{A} \mid D), \mathscr{R}^{q}\right)$ is locally contained in a subvariety of dimension $\leqq \rho$. There exists a subvariety $Z$ of dimension $\leqq \rho$ in a polydisc $W=\Delta\left(\pi(x) ; t_{1}, \ldots, t_{n-1}\right) \subset D$ such that $E^{\rho}(\mathscr{A}, \mathscr{B})$ $\cap W \subset Z$.

$$
\begin{aligned}
E^{\rho}(\mathscr{M}, \mathcal{O}) & \cap \Delta\left(x ; t_{1}, \ldots, t_{n-1}, r_{n}\right) \\
& =E^{\rho}(\mathscr{Q}, \mathscr{K}) \cap \Delta\left(x ; t_{1}, \ldots, t_{n-1}, r_{n}\right) \subset \pi^{-1}\left(E^{\rho}(\mathscr{A}, \mathscr{B}) \cap W\right) \subset \pi^{-1}(Z) .
\end{aligned}
$$

$\pi^{-1}(Z)$ is a subvariety of dimension $\leqq \rho$ in $\Delta\left(x ; t_{1}, \ldots, t_{n-1}, r_{n}\right)$. The case $p=1$ is proved.

(b) The case of a general $p \geqq 1 . \mathcal{O}^{p}=\mathcal{O}^{p-1} \oplus \mathcal{O}$. Let $\alpha: \mathcal{O}^{p} \rightarrow \mathcal{O}^{p-1}$ be the projection onto the first summand and $\beta: \mathcal{O} \rightarrow \mathcal{O}^{p}$ be the injection from the second summand. Let $\mathscr{N}=\alpha(\mathscr{M})$ and $\mathscr{P}=\mathscr{M} \cap \beta(\mathcal{O})$. Take $x \in G$. Then by induction hypothesis and by (a) there exist subvarieties $Z_{1}$ and $Z_{2}$ of dimensions $\leqq \rho$ in an open neighborhood $U$ of $x$ such that $U \cap E^{\rho}\left(\mathscr{N}, \mathcal{O}^{p-1}\right) \subset Z_{1}$ and $U \cap E^{\rho}(\mathscr{P}, \mathcal{O}) \subset Z_{2}$. It is readily checked that $U \cap E^{\rho}\left(\mathscr{M}, \mathcal{O}^{p}\right) \subset Z_{1} \cup Z_{2}$. Q.E.D.

THEOREM 3. Suppose $\mathscr{S}$ is a coherent analytic subsheaf of a coherent sheaf $\mathscr{T}$ on a complex space $(X, \mathcal{O})$ and $\rho$ is a nonnegative integer. Then $\mathscr{S}_{[\rho]}$ is coherent and $E^{\circ}(\mathscr{S}, \mathscr{T})$ is a subvariety of dimension $\leqq \rho$ in $X$.

Proof. First we prove the coherence of $\mathscr{S}_{[\rho]}$. Coherence is a local property. Take $x \in X$. By Theorem 2 there exists a subvariety $A$ of dimension $\leqq \rho$ in an open neighborhood $U$ of $x$ in $X$ such that $U \cap E^{\rho}(\mathscr{S}, \mathscr{T}) \subset A$.

Since $\operatorname{dim} A \leqq \rho, \mathscr{S}_{[\rho]} \mid U=(\mathscr{S} \mid U)[A]$. Hence $\mathscr{S}_{[\rho]} \mid U$ is coherent by Theorem 1 . $\mathscr{S}_{[\rho]}$ is coherent.

$E^{\rho}(\mathscr{S}, \mathscr{T})=E\left(\left(\mathscr{S}: \mathscr{S}_{[\rho]}\right), \mathcal{O}\right)$ is a subvariety, because $\mathscr{S}: \mathscr{S}_{[\rho]}$ is coherent. Since $E^{\rho}(\mathscr{S}, \mathscr{T})$ is locally contained in a subvariety of dimension $\leqq \rho, E^{\rho}(\mathscr{S}, \mathscr{T})$ is a subvariety of dimension $\leqq \rho$ in $X$. Q.E.D. 
Corollary. $\mathscr{S}_{[\rho]}=\mathscr{S}\left[E^{\rho}(\mathscr{S}, \mathscr{T})\right]$.

II. Noether-Lasker decomposition of subsheaves. Suppose $\mathscr{S}$ is a coherent analytic subsheaf of a coherent analytic sheaf $\mathscr{T}$ on a complex space. Let $E^{\rho}(\mathscr{S}, \mathscr{T})=\bigcup_{i \in I(\rho)} Y_{i}^{\rho}$ be the decomposition into irreducible branches. Then we call each nonempty $Y_{i}^{\rho}, \rho \geqq 0, i \in I(\rho)$, an associated subvariety of $\mathscr{S}$ in $\mathscr{T}$ and denote the set of all associated subvarieties of $\mathscr{S}$ in $\mathscr{T}$ by $\mathscr{X}(\mathscr{S}, \mathscr{T})$. From the definition we see readily that $\mathscr{X}(\mathscr{S}, \mathscr{T})$ is locally finite. $\mathscr{S}$ is called a primary subsheaf of $\mathscr{T}$ if $\mathscr{S}$ has only one associated subvariety.

The following lemma is a well-known algebraic fact [5, Appendix, Chapter IV]:

Lemma 2. Suppose $R$ is a Noetherian ring and $N$ is an $R$-submodule of a finitely generated $R$-module M. A prime ideal $P$ in $R$ is an associated prime ideal in the Noether-Lasker decomposition of $N$ as a submodule of $M$ if and only if $P=\sqrt{ }(N: f)$ for some $f \in M$.

THEOREM 4. Suppose $\mathscr{S}$ is a coherent analytic subsheaf of a coherent analytic sheaf $\mathscr{T}$ on a complex space $(X, \mathcal{O})$ and $x \in X$. Let $\left\{X_{i}^{\rho} \mid \rho \geqq 0, i \in J(\rho)\right\}$ be the set of all associated subvarieties of $\mathscr{S}$ passing through $x$, where $\operatorname{dim} X_{i}=\rho, i \in J(\rho)$, and suppose (Id $\left.X_{i}^{\rho}\right)_{x}=\bigcap_{j \in K(o, i)} P_{i j}^{p}$ is the decomposition into prime ideals. Then $\left\{P_{i j}^{\rho} \mid \rho \geqq 0, i \in J(\rho), j \in K(\rho, i)\right\}=P\left(\mathscr{S}_{x}, \mathscr{T}_{x}\right)$.

Proof. Suppose $P \in P\left(\mathscr{S}_{x}, \mathscr{T}_{x}\right)$ and $\operatorname{dim} P=\rho$. Then $P=\sqrt{ }\left(\mathscr{S}_{x}: f\right)$ for some $f \in \mathscr{T}_{x}$ by Lemma 2. $P$ defines a subvariety $V$ of dimension $\rho$ in an open neighborhood $D$ of $x$ in $X$. We can suppose after a shrinking of $D$ that there exists $g \in \Gamma(D, \mathscr{T})$ such that $g_{x}=f$ and $\operatorname{Id} V=\sqrt{ }((\mathscr{S} \mid D): g)$. This implies that

$$
\left\{y \mid y \in D, g_{y} \in \mathscr{S}_{y}\right\}=D-V .
$$

Hence $V \subset E^{\rho}(\mathscr{S}, \mathscr{T})$. Since $\operatorname{dim} V=\rho$ and $\operatorname{dim} E^{\rho}(\mathscr{S}, \mathscr{T}) \leqq \rho, P=P_{i j}^{\rho}$ for some $i \in J(\rho)$ and some $j \in K(\rho, i)$.

Fix $\rho \geqq 0$ and $i \in J(\rho)$. By definition $X_{i}^{\rho}$ is an irreducible branch of $E^{\sigma}(\mathscr{S}, \mathscr{T})$ for some $\sigma \geqq \rho$. Let $U$ be a Stein open neighborhood of $x$ in $X$ such that $U \cap X_{i}^{\rho}$ $=\bigcup_{j \in K(\rho, i)} X_{i j}^{p}$ is the decomposition into irreducible branches and $P_{i j}^{p}=\left(\operatorname{Id} X_{i j}^{p}\right)_{x}$, $j \in K(\rho, i)$. Fix $j \in K(\rho, i)$. Let $Z^{1}$ be the union of irreducible branches of $E^{\sigma}(\mathscr{S}, \mathscr{T}) \cap U$ other than $X_{i j}^{\rho}$ and let

$$
Z=Z^{1} \cup\left(E^{\rho-1}(\mathscr{S}, \mathscr{T}) \cap U\right) .
$$

Take $y \in X_{i j}^{\rho}-Z$.

$$
(\mathscr{S} \mid U)\left[X_{i j}^{\rho}\right]_{y}=\mathscr{S}_{[\sigma] y} \neq \mathscr{S}_{y} .
$$

Since $(\mathscr{S} \mid U)\left[X_{i j}^{\rho}\right]$ is generated by global sections [1, Satz 4, $\left.\S 2\right]$, there exists $t \in \Gamma\left(U,(\mathscr{S} \mid U)\left[X_{i j}^{\rho}\right]\right)$ such that $t_{y} \notin \mathscr{S}_{y}$.

Let $Y=E(\mathscr{S} \mid U,(\mathscr{S} \mid U)+(\mathcal{O} \mid U) t)$. Since $X_{i j}^{\rho}$ is irreducible, if $Y \neq X_{i j}^{\rho}$, then $\operatorname{dim} Y<\rho$ and $t_{y} \in \mathscr{S}_{[\rho-1] y}=\mathscr{S}_{y}$ (contradiction). Hence $Y=X_{i j}^{\rho} . \quad P_{i j}^{\rho}=\left(\operatorname{Id} X_{i j}^{\rho}\right)_{x}$ $=(\operatorname{Id} Y)_{x}=\sqrt{ }\left(\mathscr{S}_{x}: t_{x}\right)$. By Lemma 2, $P_{i j}^{\rho} \in P\left(\mathscr{S}_{x}, \mathscr{T}_{x}\right)$. Q.E.D. 
This theorem gives us a characterization of associated subvarieties and tells us that the subvariety-germs defined by associated prime ideals in the Noether-Lasker decomposition of the stalks of $\mathscr{S}$ can be pieced together to form global subvarieties.

Corollary 1. If $Y \in \mathscr{X}(\mathscr{S}, \mathscr{T})$, then $E(\mathscr{S}, \mathscr{S}[Y])=Y$.

Proof. Obviously $E(\mathscr{S}, \mathscr{S}[Y]) \subset Y$. Suppose $x \in Y$ and $P \in P\left((\operatorname{Id} Y)_{x}, \mathcal{O}_{x}\right)$. Then by Theorem 4, $P \in P\left(\mathscr{S}_{x}, \mathscr{T}_{x}\right)$. By Lemma $2, P=\sqrt{ }\left(\mathscr{S}_{x}: s\right)$ for some $s \in \mathscr{T}_{x}$. $s \in \mathscr{S}[Y]_{x}-\mathscr{S}_{x}$. Q.E.D.

COROLlary 2. Suppose $\mathscr{R}$ is a coherent analytic subsheaf of $\mathscr{T}$ and $\mathscr{S} \subset \mathscr{R} \subset \mathscr{T}$ and $\mathscr{X}(\mathscr{S}, \mathscr{T})=\left\{X^{i} \mid i \in I\right\}$. Then $\mathscr{X}(\mathscr{S}, \mathscr{R}) \subset \mathscr{X}(\mathscr{S}, \mathscr{T})$. Hence there is a subset $J$ of I such that $E(\mathscr{S}, \mathscr{R})=\bigcup\left\{X^{i} \mid i \in J\right\}$.

Proof. Suppose $Y \in \mathscr{X}(\mathscr{S}, \mathscr{R})$. Take $y \in Y$ and $P \in P\left((\operatorname{Id} Y)_{y}, \mathcal{O}_{y}\right)$. By Theorem 4, $P \in P\left(\mathscr{S}_{y}, \mathscr{R}_{y}\right)$. By Lemma $2, P=\sqrt{ }\left(\mathscr{S}_{y}: s\right)$ for some $s \in \mathscr{R}_{y}$. Since $s \in \mathscr{T}_{y}$, by Lemma $2, P \in P\left(\mathscr{S}_{y}, \mathscr{T}_{y}\right)$.

By Theorem 4, $P \in P\left(\left(\operatorname{Id~} X^{i}\right)_{y}, \mathcal{O}_{y}\right)$ for some $i \in I$ such that $y \in X^{i}$. Since the two irreducible subvarieties $X^{i}$ and $Y$ have a branch-germ in common at $y, X^{i}=Y$. Hence $\mathscr{X}(\mathscr{S}, \mathscr{R}) \subset \mathscr{X}(\mathscr{S}, \mathscr{T})$. The existence of $J$ follows from

$$
E(\mathscr{S}, \mathscr{R})=\bigcup\{Y \mid Y \in \mathscr{X}(\mathscr{S}, \mathscr{R})\} . \quad \text { Q.E.D. }
$$

THEOREM 5. Suppose $\mathscr{S}$ is a coherent analytic subsheaf of a coherent analytic sheaf $\mathscr{T}$ on a complex space $(X, \mathcal{O})$ and $A$ is a subvariety of $X$. Suppose $\mathscr{X}(\mathscr{S}, \mathscr{T})$ $=\left\{X^{i} \mid i \in I\right\}, x \in A$, and $I^{\prime}=\left\{i \in I \mid x \in X^{i}\right\}$. Suppose

$$
P\left(\left(\operatorname{Id} X^{i}\right)_{x}, \mathcal{O}_{x}\right)=\left\{P_{i j} \mid j \in J_{i}\right\}, \quad i \in I^{\prime} .
$$

Let $\mathscr{S}_{x}=\bigcap\left\{Q_{i j} \mid i \in I^{\prime}, j \in J_{i}\right\}$ be a Noether-Lasker decomposition of $\mathscr{S}_{x}$, where the radical of $Q_{i j}$ is $P_{i j}, i \in I^{\prime}, j \in J_{i}$, and let $K=\left\{i \mid i \in I^{\prime}, X^{i} \notin A\right\}$. Then

$$
(\mathscr{S}[A])_{x}=\bigcap\left\{Q_{i j} \mid i \in K, j \in J_{i}\right\} .
$$

Proof. Let $\mathscr{A}=\operatorname{Id} A$. By Theorem 1

$$
\begin{aligned}
\mathscr{S}[A]_{x} & =\bigcup_{n=1}^{\infty}\left(\mathscr{S}_{x}: \mathscr{A}_{x}^{n}\right)=\bigcup_{n=1}^{\infty} \bigcap\left\{Q_{i j}: \mathscr{A}_{x}^{n} \mid i \in I^{\prime}, j \in J_{i}\right\} \\
& =\bigcap\left\{\bigcup_{n=1}^{\infty}\left(Q_{i j}: \mathscr{A}_{x}^{n}\right) \mid i \in I^{\prime}, j \in J_{i}\right\} .
\end{aligned}
$$

For $i \in I^{\prime}-K, P_{i j} \supset \mathscr{A}_{x}$ and hence $Q_{i j}: \mathscr{A}_{x}^{n}=\mathscr{T}_{x}$ for $n$ sufficiently large. For $i \in K$, $P_{i j} \ngtr \mathscr{A}_{x}$ and hence $Q_{i j}: \mathscr{A}_{x}^{n}=Q_{i j}$ for every $n$. Therefore

$$
\mathscr{S}[A]_{x}=\bigcap\left\{Q_{i j} \mid i \in K, j \in J_{i}\right\} .
$$

Corollary. $\mathscr{X}(\mathscr{S}[A], \mathscr{T})=\left\{X^{i} \mid X^{i} \notin A\right\}$ and

$$
E(\mathscr{S}[A], \mathscr{T})=\bigcup\{Y \mid Y \in \mathscr{X}(\mathscr{S}, \mathscr{T}), Y \notin A\} .
$$


Proof. The first assertion follows from Theorems 4 and 5 and the second assertion follows from the first. Q.E.D.

LEMMA 3. Suppose $\mathscr{S} \subset \mathscr{R}$ are coherent analytic subsheaves of a coherent analytic sheaf $\mathscr{T}$ on a complex space $(X, \mathcal{O})$. Suppose $\mathscr{I}$ is the ideal-sheaf of $E(\mathscr{S}, \mathscr{R})$ and $x \in E(\mathscr{S}, \mathscr{R})$. Then there is a natural number $k$ such that $\left(\left(\mathscr{I}^{k} \mathscr{T}+\mathscr{S}\right) \cap \mathscr{R}\right)_{x}=\mathscr{S}_{x}$.

Proof. $E(0, \mathscr{R} / \mathscr{S})=E(\mathscr{S}, \mathscr{R})$. By Hilbert Nullstellensatz there is a natural number $l$ such that $\mathscr{I}_{x}^{l} \subset(0: \mathscr{R} / \mathscr{S})_{x}$. By the Lemma of Artin-Rees [5, Theorem 4'; $\S 2$, Chapter VIII] there exists a natural number $k$ such that $\left(\mathscr{I}^{k}(\mathscr{T} / \mathscr{S}) \cap(\mathscr{R} / \mathscr{S})\right)_{\text {x }}$ $\subset\left(\mathscr{I}^{l}(\mathscr{R} / \mathscr{S})\right)_{x}$. Hence $\left(\left(\mathscr{I}^{k} \mathscr{T}+\mathscr{S}\right) \cap \mathscr{R}\right)_{x}=\mathscr{S}_{x}$. Q.E.D.

LEMma 4. Suppose $\mathscr{S} \subset \mathscr{R}$ are coherent analytic subsheaves of a coherent analytic sheaf $\mathscr{T}$ on a complex space $(X, \mathcal{O})$. Then there exists a coherent analytic subsheaf $\mathscr{Q}$ of $\mathscr{T}$ such that $E(\mathscr{Q}, \mathscr{T})=E(\mathscr{S}, \mathscr{R})$ and $\mathscr{Q} \cap \mathscr{R}=\mathscr{S}$.

Proof. Suppose $\mathscr{X}(\mathscr{S}, \mathscr{R})=\left\{X^{i} \mid i \in I\right\}$. Let $\mathscr{I}_{i}$ be the ideal-sheaf of $X^{i}$. Take $x^{i} \in X^{i}$. By Lemma 3 and Corollary 1 to Theorem 4, there exists a natural number $k(i)$ such that

$$
\left(\left(\mathscr{I}_{i}^{k(i)} \mathscr{T}+\mathscr{S}\right) \cap \mathscr{S}\left[X^{i}\right]_{\mathscr{R}}\right)_{x^{i}}=\mathscr{S}_{x^{i}}, \quad i \in I .
$$

Since $\mathscr{X}(\mathscr{S}, \mathscr{R})$ is locally finite, $\mathscr{Q}=\bigcap_{i \in I}\left(\mathscr{I}_{i}^{k(i)} \mathscr{T}+\mathscr{S}\right)$ is a coherent analytic subsheaf of $\mathscr{T}$. Obviously $E(\mathscr{Q}, \mathscr{T}) \subset E(\mathscr{S}, \mathscr{R})$. We are going to prove that $\mathscr{Q} \cap \mathscr{R}=\mathscr{S}$.

From Corollaries 1 and 2 to Theorem 4 and (2) we conclude that

$$
E\left(\mathscr{S},\left(\mathscr{I}_{i}^{k(i)} \mathscr{T}+\mathscr{S}\right) \cap \mathscr{S}\left[X^{i}\right]_{\mathscr{R}}\right) \subset \bigcup\left\{X^{j} \mid j \in I, X^{j} \varsubsetneqq X^{i}\right\}, \quad i \in I .
$$

Obviously $\mathscr{S} \subset \mathscr{Q} \cap \mathscr{R}$. Let $Y=E(\mathscr{S}, \mathscr{Q} \cap \mathscr{R})$. By Corollary 2 to Theorem 4 there exists a subset of $J$ of $I$ such that $Y=\bigcup\left\{X^{i} \mid i \in J\right\}$. Suppose $Y \neq \varnothing$. Then take $y \in Y$. Take a relatively compact open neighborhood of $U$ of $y$ in $X$. Let $F=\left\{i \mid i \in J, X^{i} \cap U \neq \varnothing\right\} . F$ is a finite set. Take $i \in F$ such that

$$
\operatorname{dim} X^{i}=\max \left\{\operatorname{dim} X^{j} \mid j \in F\right\} .
$$

Take an open neighborhood $W$ of a point $z$ of $X^{i}$ in $U$ such that

$$
W \cap\left(\bigcup\left\{X^{j} \mid j \in I, X^{j} \ngtr X^{i}\right\}\right)=\varnothing .
$$

$Y \cap W=X^{i} \cap W .(\mathscr{Q} \cap \mathscr{R})\left|W \subset \mathscr{S}\left[X^{i}\right]_{\mathscr{R}}\right| W$. By (3) $\left(\mathscr{I}_{i}^{k(i)} \mathscr{T}+\mathscr{S}\right) \cap \mathscr{S}\left[X^{i}\right]_{\mathscr{R}} \mid W=$ $\mathscr{S} \mid W$. Hence

$$
\mathscr{S}|W \subset \mathscr{Q} \cap \mathscr{R}| W \subset\left(\mathscr{I}_{i}^{k(i)} \mathscr{T}+\mathscr{S}\right) \cap \mathscr{S}\left[X^{i}\right]_{\mathscr{R}}|W=\mathscr{S}| W .
$$

$z \notin Y$ (contradiction). Hence $Y=\varnothing$ and $\mathscr{S}=\mathscr{Q} \cap \mathscr{R}$.

Suppose $E(\mathscr{Q}, \mathscr{T}) \neq E(\mathscr{S}, \mathscr{R})$. Take $x \in E(\mathscr{S}, \mathscr{R})-E(\mathscr{Q}, \mathscr{T})$. Then $\mathscr{2}_{x}=\mathscr{T}_{x}$. $\mathscr{S}_{x}=\mathscr{Q}_{x} \cap \mathscr{R}_{x}=\mathscr{R}_{x}$, contradicting that $x \in E(\mathscr{S}, \mathscr{R})$. Hence $E(\mathscr{Q}, \mathscr{T})=E(\mathscr{S}, \mathscr{R})$. Q.E.D. 
LEMMA 5. Suppose $\mathscr{S}$ is a coherent analytic subsheaf of a coherent analytic sheaf $\mathscr{T}$ on a complex space $(X, \mathcal{O})$ and $\mathscr{X}(\mathscr{S}, \mathscr{T})=\left\{X^{i} \mid i \in I\right\}$. Let $J=\left\{i \mid i \in I, X^{i}\right.$ is maximal in $\mathscr{X}(\mathscr{S}, \mathscr{T})\}$. Then there exist a coherent analytic subsheaf $\mathscr{R}$ of $\mathscr{T}$ and primary subsheaves $\mathscr{Q}_{i}$ of $\mathscr{T}, i \in J$, such that (i) $E(\mathscr{R}, \mathscr{T})=\bigcup_{i \in K} X^{i}$, where $K=I-J$, (ii) $E\left(\mathscr{Q}_{i}, \mathscr{T}\right)=X^{i}, i \in J$, and (iii) $\left(\bigcap_{i \in J} \mathscr{Q}_{i}\right) \cap \mathscr{R}=\mathscr{S}$.

Proof. For $i \in J$ let $Y^{i}=\bigcup\left\{X^{j} \mid j \in I\right.$ and $\left.j \neq i\right\}$ and define $\mathscr{Q}_{i}=\mathscr{S}\left[Y^{i}\right]$.

Then by Corollary to Theorem $5,\left\{X^{i}\right\}=\mathscr{X}\left(\mathscr{Q}_{i}, \mathscr{T}\right), i \in J$. Hence $E\left(\mathscr{Q}_{i}, \mathscr{T}\right)=X^{i}$ and $\mathscr{Q}_{i}$ is a primary subsheaf of $\mathscr{T}, i \in J$. Take $y^{i} \in X^{i}-Y^{i}, i \in J$. Then $\left(\mathscr{Q}_{i}\right)_{y^{i}}=\mathscr{S}_{y^{i}}$, $i \in J$. Since $\mathscr{Q}_{i} \supset \mathscr{S}, i \in J$, we have

$$
\left(\bigcap_{j \in J} \mathscr{Q}_{j}\right)_{y^{i}}=\mathscr{S}_{y^{4}}, \quad i \in J .
$$

Since $\mathscr{X}(\mathscr{S}, \mathscr{T})$ is locally finite, $\bigcap_{i \in J} \mathscr{Q}_{i}$ is a coherent analytic subsheaf of $\mathscr{T}$. By (4) and Corollary 2 to Theorem $4 E\left(\mathscr{S}, \bigcap_{i \in J} \mathscr{Q}_{i}\right) \subset \bigcup_{i \in K} X^{i}$.

Suppose $x \in \bigcup_{i \in K} X^{i}-E\left(\mathscr{S}, \bigcap_{i \in J} \mathscr{Q}_{i}\right)$. Then $x \in X^{j}$ for some $j \in K$ and $\mathscr{S}_{x}=\bigcap_{i \in J}\left(\mathscr{Q}_{i}\right)_{x}$. Let $L=\left\{i \mid i \in J, x \in X^{i}\right\}$. Since

$$
\begin{aligned}
P\left(\left(\mathscr{Q}_{i}\right)_{x}, \mathscr{T}_{x}\right) & =P\left(\left(\operatorname{Id} X^{i}\right)_{x}, \mathcal{O}_{x}\right), \quad i \in L, \\
P\left(\mathscr{S}_{x}, \mathscr{T}_{x}\right) & \subset \bigcup_{i \in L} P\left(\left(\operatorname{Id} X^{i}\right)_{x}, \mathcal{O}_{x}\right) .
\end{aligned}
$$

Since

$$
P\left(\left(\operatorname{Id} X^{j}\right)_{x}, \mathcal{O}_{x}\right) \cap\left(\bigcup_{i \in L} P\left(\left(\operatorname{Id} X^{i}\right)_{x}, \mathcal{O}_{x}\right)\right)=\varnothing,
$$

Theorem 4, which asserts that $P\left(\left(\operatorname{Id~} X^{j}\right)_{x}, \mathcal{O}_{x}\right) \subset P\left(\mathscr{S}_{x}, \mathscr{T}_{x}\right)$, is contradicted. Hence $E\left(\mathscr{S}, \bigcap_{i \in J} \mathscr{Q}_{i}\right)=\bigcup_{i \in K} X^{i}$. By Lemma 4 there exists a coherent analytic subsheaf $\mathscr{R}$ of $\mathscr{T}$ such that $\left(\bigcap_{i \in J} \mathscr{Q}_{i}\right) \cap \mathscr{R}=\mathscr{S}$ and $E(\mathscr{R}, \mathscr{T})=\bigcup_{i \in K} X^{i}$. Q.E.D.

THEOREM 6 (NOETHER-LASKER DECOMPOSITION OF COHERENT SUBHEAVES). Suppose $\mathscr{S}$ is a coherent analytic subsheaf of a coherent analytic sheaf $\mathscr{T}$ on a complex space $(X, \mathcal{O})$ and $\mathscr{X}(\mathscr{S}, \mathscr{T})=\left\{X^{i} \mid i \in I\right\}$. Then for every $i \in I$, there exists a primary subsheaf $\mathscr{Q}_{i}$ of $\mathscr{T}$ such that $E\left(\mathscr{Q}_{i}, \mathscr{T}\right)=X^{i}$ and $\bigcap_{i \in I} \mathscr{Q}_{i}=\mathscr{S}$.

Proof. For $Y \in \mathscr{X}(\mathscr{S}, \mathscr{T})$ define the depth of $Y$ in $\mathscr{X}(\mathscr{S}, \mathscr{T})$ to be $\sup \{l \mid$ there exist $Y_{j} \in \mathscr{X}(\mathscr{S}, \mathscr{T}), 0 \leqq j \leqq l$, such that $Y_{0}=Y$ and $Y_{j} \varsubsetneqq Y_{j+1}$ for $\left.0 \leqq j<l\right\}$. If $Y_{j} \in \mathscr{X}(\mathscr{S}, \mathscr{T}), 0 \leqq j \leqq l$, and $Y_{j} \varsubsetneqq Y_{j+1}, 0 \leqq j<l$, then for $x \in Y_{0}, \operatorname{dim}_{x} Y_{j}<\operatorname{dim}_{x} Y_{j+1}$ (because $Y_{i}$ is irreducible) and $l \leqq \operatorname{dim}_{x} X$. So the depth of $Y$ in $\mathscr{X}(\mathscr{S}, \mathscr{T})$ is finite for $Y \in \mathscr{X}(\mathscr{S}, \mathscr{T})$. For $i \in I$ denote the depth of $X^{i}$ by $d_{i}$, and, for any nonnegative integer $d$, let $I_{d}=\left\{i \mid i \in I d_{i}=d\right\}, J_{d}=\bigcup_{l \leqq d} I_{l}$, and $K_{d}=I-J_{d}$.

We are going to prove by induction on $d$ the following:

(5) For every $d \geqq 0$ there exist primary subsheaves $\mathscr{Q}_{i}$ of $\mathscr{T}$ for $i \in I_{d}$ and a coherent analytic subsheaf $\mathscr{R}_{d}$ of $\mathscr{T}$ such that (i) $E\left(\mathscr{Q}_{i}, \mathscr{T}\right)=X^{i}, i \in I_{d}$, (ii) $E\left(\mathscr{R}_{d}, \mathscr{T}\right)=\bigcup_{i \in K_{d}} X^{i}$, and (iii) $\left(\bigcap_{i \in J_{d}} \mathscr{Q}_{i}\right) \cap \mathscr{R}_{d}=\mathscr{S}$. 
$J_{0}=\left\{X^{i} \mid X^{i}\right.$ is maximal in $\left.\mathscr{X}(\mathscr{S}, \mathscr{T})\right\}$. By Lemma $5,(5)$ is true for $d=0$. Suppose (5) is proved for $0 \leqq d \leqq e$. Since $E\left(\mathscr{R}_{e}, \mathscr{T}\right)=\bigcup_{i \in K_{e}} X^{i}$ and

$$
E\left(\mathscr{R}_{e}, \mathscr{T}\right)=\bigcup\left\{Y \mid Y \in \mathscr{X}\left(\mathscr{R}_{e}, \mathscr{T}\right)\right\},
$$

$\left\{X^{i} \mid i \in I_{e+1}\right\}=\left\{Y \in \mathscr{X}\left(\mathscr{R}_{e}, \mathscr{T}\right) \mid Y\right.$ is maximal in $\left.\mathscr{X}\left(\mathscr{R}_{e}, \mathscr{T}\right)\right\}$. By Lemma 5 there exist primary subsheaves $\mathscr{Q}_{i}$ of $\mathscr{T}, i \in I_{e+1}$, and a coherent analytic subsheaf $\mathscr{B}$ of $\mathscr{T}$ such that (i) $E\left(\mathscr{Q}_{i}, \mathscr{T}\right)=X^{i}, i \in I_{e+1}$, (ii) $E(\mathscr{B}, \mathscr{T})$ is thin in $\bigcup_{i \in I_{e+1}} X^{i}$, and (iii) $\left(\bigcap_{i \in I_{e+1}} \mathscr{Q}_{i}\right) \cap \mathscr{B}=\mathscr{R}_{e}$. Hence $\left(\bigcap_{i \in J_{e+1}} \mathscr{Q}_{i}\right) \cap \mathscr{B}=\mathscr{S}$. Let

$$
Z=\left\{Y \mid Y \in \mathscr{X}(\mathscr{B}, \mathscr{T}), \quad Y \notin \bigcup_{i \in K_{e+1}} X^{i}\right\}
$$

By Corollary to Theorem $5, E\left(\mathscr{B}[Z]_{\mathscr{F}}, \mathscr{T}\right) \subset \bigcup_{i \in K_{e+1}} X^{i}$. Let

$$
V=E\left(\mathscr{S},\left(\bigcap_{i \in J_{e+1}} \mathscr{Q}_{i}\right) \cap \mathscr{B}[Z]_{\mathscr{T}}\right) .
$$

Then $V \subset Z$. Since $Z$ is thin in $\bigcup_{t \in I_{e+1}} X^{i}$, by Corollary 2 to Theorem 4, $V \subset \bigcup_{i \in K_{e+1}} X^{i}$.

By Lemma 4 there exists a coherent analytic subsheaf $\mathscr{H}$ of $\mathscr{T}$ such that $E(\mathscr{H}, \mathscr{T})=V$ and $\left(\bigcap_{i \in J_{e+1}} \mathscr{Q}_{i}\right) \cap \mathscr{B}[Z]_{\mathscr{T}} \cap \mathscr{H}=\mathscr{S}$. Let $\mathscr{R}_{e+1}=\mathscr{B}[Z]_{\mathscr{g}} \cap \mathscr{H}$. Then $\left(\bigcap_{i \in J_{e+1}} \mathscr{Q}_{i}\right) \cap \mathscr{R}_{e+1}=\mathscr{S}$ and $E\left(\mathscr{R}_{e+1}, \mathscr{T}\right) \subset \bigcup_{i \in K_{e+1}} X^{i}$. Suppose $E\left(\mathscr{R}_{e+1}, \mathscr{T}\right)$ $\neq \bigcup_{i \in K_{e+1}} X^{i}$. Take $x \in \bigcup_{i \in K_{e+1}} X^{i}-E\left(\mathscr{R}_{e+1}, \mathscr{T}\right) . x \in X^{j}$ for some $j \in K_{e+1}$ and $\mathscr{S}_{x}=\bigcap_{i \in J_{e+1}}\left(\mathscr{Q}_{i}\right)_{x}$. Let $L=\left\{i \mid i \in J_{e+1}, x \in X^{i}\right\}$. Since $P\left(\left(\mathscr{Q}_{i}\right)_{x}, \mathscr{T}_{x}\right)=P\left(\left(\operatorname{Id} X^{i}\right)_{x}, \mathcal{O}_{x}\right)$, $i \in L, P\left(\mathscr{S}_{x}, \mathscr{T}_{x}\right) \subset \bigcup_{i \in L} P\left(\left(\operatorname{Id~} X^{i}\right)_{x}, \mathcal{O}_{x}\right)$. However,

$$
P\left(\left(\operatorname{Id} X^{j}\right)_{x}, \mathcal{O}_{x}\right) \cap\left(\bigcup_{i \in L} P\left(\left(\operatorname{Id} X^{i}\right)_{x}, \mathcal{O}_{x}\right)\right)=\varnothing,
$$

contradicting Theorem 4, which asserts $P\left(\left(\operatorname{Id} X^{j}\right)_{x}, \mathcal{O}_{x}\right) \subset P\left(\mathscr{S}_{x}, \mathscr{T}_{x}\right)$. Hence $E\left(\mathscr{R}_{e+1}, \mathscr{T}\right)=\bigcup_{i \in K_{e+1}} X^{i}$. The induction process is complete and (5) is proved.

We claim that $\mathscr{S}=\bigcap_{i \in I} \mathscr{Q}_{i}$. Obviously $\mathscr{S} \subset \bigcap_{i \in I} \mathscr{Q}_{i}$. Take $x \in X$.

$$
F=\left\{i \mid i \in I, x \in X^{i}\right\}
$$

is a finite set. Take $d \geqq \max \left\{d_{i} \mid i \in F\right\}$. Then $x \notin \bigcup_{i \in K_{d}} X^{i} .\left(\mathscr{R}_{d}\right)_{x}=\mathscr{T}_{x}$.

$$
\mathscr{S}_{x}=\left(\bigcap_{i \in J_{d}}\left(\mathscr{Q}_{i}\right)_{x} \cap\left(\mathscr{R}_{d}\right)\right)_{x}=\bigcap_{i \in J_{d}}\left(\mathscr{Q}_{i}\right)_{x} \supset \bigcap_{i \in I}\left(\mathscr{Q}_{i}\right)_{x} \text {. Q.E.D. }
$$

Remark. The decomposition $\mathscr{S}=\bigcap_{i \in I} \mathscr{Q}_{i}$ is irredundant, i.e. $\mathscr{S} \neq \bigcap_{i \in I-\{j\}} \mathscr{Q}_{i}$ for any $j \in I$; for otherwise by Theorem 4 we have

$$
\mathscr{X}(\mathscr{S}, \mathscr{T}) \subset \bigcup_{i \in I-\{j\}} \mathscr{X}\left(\mathscr{Q}_{i}, \mathscr{T}\right)=\left\{X^{i} \mid i \in I-\{j\}\right\} .
$$

In general, $\mathscr{Q}_{i}, i \in I$, is not uniquely determined. For example, when $(X, \mathcal{O})$ is $C^{2}$ with coordinate-functions $z_{1}$ and $z_{2}$, then $\left(O z_{1}\right) \cap\left(O z_{1}^{2}+O z_{2}\right)=\left(O z_{1}\right) \cap\left(O z_{1}+O z_{2}\right)^{2}$ are two different irredundant Noether-Lasker decompositions. 
However, corresponding to the uniqueness of isolated ideal components in the usual Noether-Lasker decomposition in rings, we have the following:

A subset $L$ of $\mathscr{X}(\mathscr{S}, \mathscr{T})$ is called an isolated system of associated subvarieties if $Y_{1} \subset Y_{2}, Y_{i} \in \mathscr{X}(\mathscr{S}, \mathscr{T}), i=1,2$, and $Y_{1} \in L$ imply $Y_{2} \in L$. If $L$ is an isolated system of associated subvarieties, then $\bigcap\left\{\mathscr{Q}_{i} \mid X^{i} \in L\right\}$ is unique, because it is equal to $\mathscr{S}\left[\bigcup\left\{X^{i} \mid X^{i} \notin L\right\}\right]$ by Corollary to Theorem 5 .

THEOREM 7. Suppose $\mathscr{S}$ is a coherent analytic subsheaf of a coherent analytic sheaf $\mathscr{T}$ on a complex space $(X, \mathcal{O})$. If $\mathscr{S}$ is primary, then $\Gamma(X, \mathscr{S})$ is a primary $\Gamma(X, \mathcal{O})$-submodule of $\Gamma(X, \mathscr{T})$. The converse is true if $(X, \mathcal{O})$ is Stein and $\Gamma(X, \mathscr{S})$ $\neq \Gamma(X, \mathscr{T})$.

Proof. (i) Suppose $\mathscr{S}$ is primary. Let $\{Y\}=\mathscr{X}(\mathscr{S}, \mathscr{T})$. We are going to prove that $\Gamma(X, \mathscr{S})$ is a primary $\Gamma(X, \mathcal{O})$-submodule of $\Gamma(X, \mathscr{T})$ with $\Gamma(X$, Id $Y)$ as its radical.

Take $f \in \Gamma(X$, Id $Y)$. Fix $y \in Y$. Since $E(\mathscr{S}: \mathscr{T}, \mathcal{O})=Y$, by Hilbert Nullstellensatz $f_{y}^{k} \mathscr{T}_{y} \subset \mathscr{S}_{y}$ for some natural number $k . \mathscr{X}\left(\mathscr{S}, f^{k} \mathscr{T}+\mathscr{S}\right) \subset\{Y\}$ by Corollary 2 to Theorem 4. $y \notin E\left(\mathscr{S}, f^{k} \mathscr{T}+\mathscr{S}\right)$ implies $E\left(\mathscr{S}, f^{k} \mathscr{T}+\mathscr{S}\right)=\varnothing \cdot f^{k} \Gamma(X, \mathscr{T}) \subset \Gamma(X, \mathscr{S})$.

Suppose $g \in \Gamma(X, \mathcal{O})-\Gamma(X$, Id $Y)$ and $s \in \Gamma(X, \mathscr{T})$ such that $g s \in \Gamma(X, \mathscr{S})$. For some $y \in Y g$ does not vanish at $y$. Then $s_{y} \in \mathscr{S}_{y} . \mathscr{X}(\mathscr{S}, \mathcal{O} s+\mathscr{S}) \subset\{Y\}$ by Corollary 2 to Theorem 4. $y \notin E(\mathscr{S}, \mathcal{O} s+\mathscr{S})$ implies $E(\mathscr{S}, \mathcal{O} s+\mathscr{T})=\varnothing . s \in \Gamma(X, \mathscr{T})$.

(ii) Suppose $\Gamma(X, \mathscr{S})$ is a primary $\Gamma(X, \mathcal{O})$-submodule of $\Gamma(X, \mathscr{T})$. Suppose $\Gamma(X, \mathscr{S}) \neq \Gamma(X, \mathscr{T})$ and $(X, \mathcal{O})$ is Stein. Let $P \subset \Gamma(X, \mathcal{O})$ be the radical of $\Gamma(X, \mathscr{S})$. $P$ defines a subvariety $Y$ in $X$. Clearly $E(\mathscr{S}, \mathscr{J}) \subset Y$ and $E(\mathscr{S}, \mathscr{J}) \neq \varnothing$.

We claim $P=\Gamma(X$, Id $Y)$. Take $f \in \Gamma(X$, Id $Y)$. Fix $y \in Y$. By Hilbert Nullstellensatz $f_{y}^{k} \in\left(\sum_{i=1}^{l} \mathcal{O} g_{i}\right)_{y}$ for some natural number $k$ and some $g_{1}, \ldots, g_{l} \in P$. $y \notin E\left(\left(\sum_{i=1}^{l} \mathcal{O} g_{i}\right): f, \mathcal{O}\right)$. By Cartan Theorem A there exists $h \in \Gamma\left(X,\left(\sum_{i=1}^{l} \mathcal{O} g_{i}\right): f\right)$ such that $h$ does not vanish at $y . h f \in \Gamma\left(X, \sum_{i=1}^{l} \mathcal{O} g_{i}\right)$ and $h \notin P$. Let $\varphi: \mathcal{O}^{l} \rightarrow \sum_{i=1}^{l} \mathcal{O} g_{i}$ be the sheaf-epimorphism defined by $\varphi\left(t_{1}, \ldots, t_{l}\right)=\sum_{i=1}^{l} t_{i}\left(g_{i}\right)_{x}$ for $x \in X$ and $\left(t_{1}, \ldots, t_{l}\right) \in \mathcal{O}_{x}^{p}$. Since $H^{1}(X, \operatorname{Ker} \varphi)=0$ by Cartan Theorem B, $h f=\sum_{i=1}^{l} c_{i} g_{i}$ for some $c_{1}, \ldots, c_{l} \in \Gamma(X, \mathcal{O}) . h f \in P . h \notin P$ implies $f \in P$. Hence $P=\Gamma(X$, Id $Y)$.

$Y$ is irreducible, for otherwise $Y=Y_{1} \cup Y_{2}$ for some subvarieties $Y_{1} \neq Y$ and $Y_{2} \neq Y$ and $P=\Gamma(X$, Id $Y)=\Gamma\left(X\right.$, Id $\left.Y_{1}\right) \cap \Gamma\left(X\right.$, Id $\left.Y_{2}\right)$ with $\Gamma\left(X\right.$, Id $\left.Y_{i}\right) \neq$ $\Gamma(X$, Id $Y), i=1,2$.

Suppose $Z \in \mathscr{X}(\mathscr{S}, \mathscr{T}) . Z \subset Y$. Suppose $Y \neq Z$. Then take $y \in Y-Z$. By Corollary 1 to Theorem 4, $E(\mathscr{S}: \mathscr{S}[Z], \mathcal{O})=Z$. By Cartan Theorem A there exists $f \in$ $\Gamma(X, \mathscr{S}: \mathscr{S}[Z])$ such that $f$ does not vanish at $y$ and there exists $s \in \Gamma(X, \mathscr{S}[Z])-$ $\Gamma(X, \mathscr{S})$. Hence $f \notin P, s \notin \Gamma(X, \mathscr{S})$, and $f s \in \Gamma(X, \mathscr{S})$. Contradiction. $\mathscr{X}(\mathscr{S}, \mathscr{T})$ $=\{Y\} . \mathscr{S}$ is primary. Q.E.D.

REMARKS. (i) Theorem 7 justifies the term primary subsheaf.

(ii) Theorem ' of [3] follows from Theorems 6 and 7.

The author wishes to thank Professor Robert C. Gunning for his suggestions and encouragements. 


\section{REFERENCES}

1. H. Grauert, Ein Theorem der analytischen Garbentheorie und die Modulräume komplexer Strukturen, Inst. Hautes Études Sci., No. 5, Paris, 1960.

2. R. C. Gunning and H. Rossi, Analytic functions of several complex variables, PrenticeHall, Englewood Cliffs, N. J., 1965.

3. O. Forster, Primärzelegung in Steinschen Algebren, Math. Ann. 154 (1964), 307-329.

4. W. Thimm, Lückengarben von kohärenten analytischen Modulgarben, Math. Ann. 148 (1962), 372-394.

5. O. Zariski and P. Samuel, Commutative algebra, Van Nostrand, Princeton, N. J., 1958.

Purdue University,

LAFAYETTE, INDIANA

UNIVERSITY OF NOTRE DAME,

Notre DAME, INDIANA 